Proceedings of the Operational Research Society Simulation Workshop 2021 (SW21)

M. Fakhimi, D. Robertson, and T. Boness, eds.

DOI: https://doi.org/10.36819/SW21.038

\title{
DYNAMIC COALITIONS IN COMPLEX TASK ENVIRONMENTS: TO CHANGE OR NOT TO CHANGE A WINNING TEAM?
}

\author{
Darío Blanco-Fernández \\ University of Klagenfurt \\ Klagenfurt, Austria \\ dario.blanco@aau.at
}

\section{Stephan Leitner}

University of Klagenfurt

Klagenfurt, Austria

stephan.leitner@aau.at

\author{
Alexandra Rausch \\ University of Klagenfurt \\ Klagenfurt, Austria \\ alexandra.rausch@aau.at
}

\begin{abstract}
Decision-makers are often confronted with complex tasks which cannot be solved by an individual alone but require collaboration in the form of a coalition. Previous literature argues that instability, in terms of the re-organization of a coalition concerning its members over time, is detrimental to performance. Other lines of research, such as the dynamic capabilities framework, challenge this view. Our objective is to understand the effects of instability on the performance of coalitions formed to solve complex tasks. To do so, we adapt the $N K$-model to the context of human decision-making in coalitions and introduce an auction-based mechanism for autonomous coalition formation and a learning mechanism for human agents. Preliminary results suggest that reorganizing innovative and well-performing teams is beneficial, but this is true only in certain situations.
\end{abstract}

\section{Keywords:}

self-organization, dynamic capabilities, complex and adaptive systems

\section{INTRODUCTION}

Decision-makers are often confronted with complex tasks, i.e., tasks composed of a certain number of (often highly) interrelated sub-tasks (Giannoccaro et al. 2018). Complex tasks are present in our everyday life, and can, for example, be found in the context of business operations, vaccine development, and construction projects, among many others. Such tasks' innate complexity makes it necessary for individuals to coordinate their efforts and to share their capabilities with other individuals to solve these tasks (Simon 1957). We refer to grouping up into teams as coalition formation and exclusively focus on coalitions of human decision-makers.

Previous research has identified stability (in terms of not allowing for the autonomous re-organization of coalitions over time) as a desirable characteristic of coalitions (Hsu et al. 2016). It is argued that stable coalitions come up with solutions to complex tasks associated with a higher coalition performance than the solutions of other, more unstable counterparts (Hsu et al. 2016). This is reflected in the motto never change a winning team. However, other lines of research argue that the stability in the composition of a coalition does not ensure that the coalition comes up with better-performing solutions (see, for example, Hennet and Mahjoub (2011) and Sless et al. (2018)). Given these mutually incompatible findings, it is, thus, unclear whether allowing for the autonomous re-organization of coalitions over time with different frequencies has a negative or a positive effect on performance. Can change make a winning team even better? 
Our objective is to contribute to the literature of coalitions confronted with complex decision-making tasks by gaining insights into (i) the dynamics of coalition formation and (ii) the interrelations between the frequency of coalition re-organization over time and performance. The complex task environment is based on the $N K$-model for organizational decision-making (Levinthal 1997, Leitner and Wall 2015, Wall and Leitner 2020). Within this framework, we operationalize instability as the possibility to replace the members of a coalition over time and consider different frequencies at which such a replacement can take place. Coalitions that are allowed to replace their members more (less) frequently are regarded as being relatively more unstable (stable). We observe the solutions to the complex decision-making problem that the coalitions come up with and record the associated performances. Our approach also includes autonomous coalition formation, i.e., we allow human decision-makers to form coalitions by employing a mechanism based on a second-price auction, which is a standard method for self-organization in the field of Robotics (Rizk et al. 2019).

The remainder of this paper is organized as follows: Section 2 places our research endeavor in the context of the relevant literature. In Sec. 3, we introduce the agent-based model. The results of the simulation study are presented in Sec. 4. Finally, Sec. 5 discusses the results and provides some conclusions and an outlook on future research avenues.

\section{RELATED LITERATURE}

Coalition formation has been extensively studied in the field of Economics, see, for example, Banerjee et al. (2001) and İnal (2019). Research in Economics is particularly concerned with the allocation of a coalition's performance among its members, so that the members do not have incentives to exit a coalition and join another one (i.e., they put stability in the focus) (Banerjee et al. 2001, İnal 2019). A set of solution concepts, namely, the core, the kernel, the nucleolus and the Shapley value are studied to assure stability (Tremewan and Vanberg 2016).

Since our objective is not to find an allocation mechanism that ensures a stable coalition, our research more likely connects with previous work on task allocation in the field of Robotics. Research in this field has extensively studied the allocation of sub-tasks to autonomous robotic entities (such as drones which jointly carry out a specific task) as a mean for coalition formation. In this field, the objective is to find mechanisms that improve the efficiency of solving a task; frequently used mechanisms for coalition formation which have been shown to improve this efficiency are auction-based methods (Rizk et al. 2019).

Task allocation has also been studied in the field of Managerial Science, although not as extensively as in Robotics. The ultimate goal in this area is to understand how task decomposition into sub-tasks can improve performance. Researchers have frequently made use of the $N K$-framework for modelling task allocation and decision-making in agent-based systems (Rivkin and Siggelkow 2003, Wall 2018). Note, however, that instability is often not explicitly modelled in this line of research, as it is implicitly regarded to have negative effects on performance (Hsu et al. 2016).

The dynamic capabilities framework (Teece et al. 1997) provides a theoretical framework to investigate the potential effects of the (more or less frequent) re-organization of the coalition on performance. According to this framework, both the individual decision-makers (i.e., at the microlevel) and the coalition as a collective of decision-makers (i.e., at the macro-level) must constantly adapt to the environment to improve performance (Eisenhardt and Martin 2000). The environment to which individuals and the coalition adapt may be represented by the coalition's task environment, like in our case. The task environment is based on the $N K$-framework and is shaped by the complexity of the task that the coalition faces (Giannoccaro et al. 2018). ${ }^{1}$ We follow the dynamic capabilities framework and conceptualize capabilities (which are subject to adaptation) at two levels:

- First, individuals have some capabilities to complete tasks, in terms of known actions. Each individual learns new or forgets existing actions over time to adapt their capabilities to the environment. We interpret this as the adaptation of capabilities process at the individual level.

- Second, coalitions are formed by individual agents. Over time, some members of a coalition may be replaced (in a self-organized manner), which we interpret as the adaptation at the coalition level.

\footnotetext{
${ }^{1}$ Details are provided in Sec. 3 .
} 
Following the dynamic capabilities framework, individuals and coalitions are regarded to be successful when they efficiently adapt to the environment in which they operate and, consequently, achieve higher performance levels. Having said this, the perspectives taken by the traditional economic view and the dynamic capabilities framework are mutually incompatible: The former regards stability in the composition of a coalition as beneficial (Hsu et al. 2016), whereas the latter regards the adaptation at all levels as the key to higher performance (Eisenhardt and Martin 2000).

\section{THE MODEL}

Overview Our research differs in several aspects from previous approaches that study coalition formation and task allocation in Economics, Robotics and Management Science. Regarding Economics, previous research has considered multiple coalitions at the same time and has focused on the mechanisms that prevent agents from switching between coalitions (see, for example, Banerjee et al. (2001) and İnal (2019)). Agents are usually allowed to freely leave or join the coalition (Banerjee et al. 2001, İnal 2019). In contrast, we focus on only one coalition and investigate the dynamics emerging within this coalition. We implement coalition formation as a self-organized process that follows a second-price auction and we endogenously define whether and how often the agents are allowed to join or leave a coalition. Furthermore, we model the agents in a way that they always have an incentive to participate in the coalition.

Research on coalition formation in Robotics is usually concerned with solving complex physical tasks by nonhuman entities (Rizk et al. 2019). However, we focus on coalitions formed by human decision-makers who have limited cognitive capabilities and face a complex decision-making task.

Finally, the interrelation of task allocation as a mean for coalition formation has not been extensively addressed in Management Science (Rivkin and Siggelkow 2003, Wall 2018). Moreover, the dynamic capabilities framework has not been properly related to coalition formation and task allocation literature. We contribute to this literature by explicitly addressing dynamic coalition formation and investigating the dynamics emerging from coalition formation processes in coalitions in which capabilities at both the individual and the collective level are subject to adaptation.

The task environment The complex task is modelled as a vector $\mathbf{d}_{\mathbf{t}}=\left(d_{1 t} \ldots d_{N t}\right)$ consisting of $N$ binary decisions, with $K$ inter-dependencies among decisions. This follows the $N K$-framework for organizational decision-making, which was first introduced by Levinthal (1997). As decisions are binary, there exist $2^{N}$ solutions to the decision-making problem, each with an associated performance. The mapping of each solution to its associated performance is referred to as the performance landscape. At every time step $t=1, \ldots, T$ a coalition makes decisions $d_{i t} \in\{0,1\}$ and each decision contributes $c_{i t}$ to coalition performance $C\left(\mathbf{d}_{\mathbf{t}}\right)$. Performance contributions follow a uniform distribution $c_{i t} \sim U(0,1)$, whereby each contribution $c_{i t}$ is affected by decision $d_{i t}$ and $K$ other decisions. The latter are denoted by $\overline{\mathbf{d}}_{i t}=\left(d_{j_{1}} \ldots d_{j_{K} t}\right)$, where $i, j=1, \ldots, N,\left\{j_{1}, \ldots, j_{N}\right\} \subseteq\{1, \ldots, i-1, i+1, \ldots, N\}$ and $0 \leq K \leq N-1$. Performance contributions are formalized by

$$
c_{i t}=f\left(d_{i t} ; \overline{\mathbf{d}}_{i t}\right) .
$$

Coalition performance is computed according to $C\left(\mathbf{d}_{\mathbf{t}}\right)=\frac{1}{N} \sum_{i=1}^{i=n} c_{i t}$. The parameter $K$ shapes the complexity of the decision problem and, consequently, the ruggedness of the resulting performance landscape. Once a coalition is formed, it moves in the performance landscape following a hill-climbingbased search process for a solution that has a better associated performance (Levinthal 1997).

We set $N=12$ and divide the entire decision problem into three sub-problems denoted by $N_{s}$, each consisting of four binary decisions; $s=(1 \ldots S)$ indicates the areas of expertise that refer to the sub-problems. For each of the partial binary decision problems, there exist $4^{2}$ solutions. These solutions represent capabilities within an area of expertise. The coalition strategy is the set of decisions made or the concatenation of solutions to each sub-problem (i.e., in each area of expertise), respectively, for a particular time step.

Concerning $K$, we consider three different levels of inter-dependencies, being $K=3, K=5$ and $K=11$, and referred to as a low-, mid-, and high level of inter-dependencies, respectively. We also control for the structure of the inter-dependencies, distinguishing between three particular cases: 
- In the concentrated scenario, each decision is inter-dependent with decisions of the same area of expertise or with decisions close to it (represented by the matrices under the headline concentrated in Fig. 1).

- In the scattered scenario, decisions are inter-dependent with decisions that are not in the same area of expertise (represented by the matrices under the headline scattered in Fig. 1).

- In the scenario with the maximum level of inter-dependencies, all decisions are inter-dependent with each other (represented by the matrix under the headline $K=11$ full in Fig. 1).

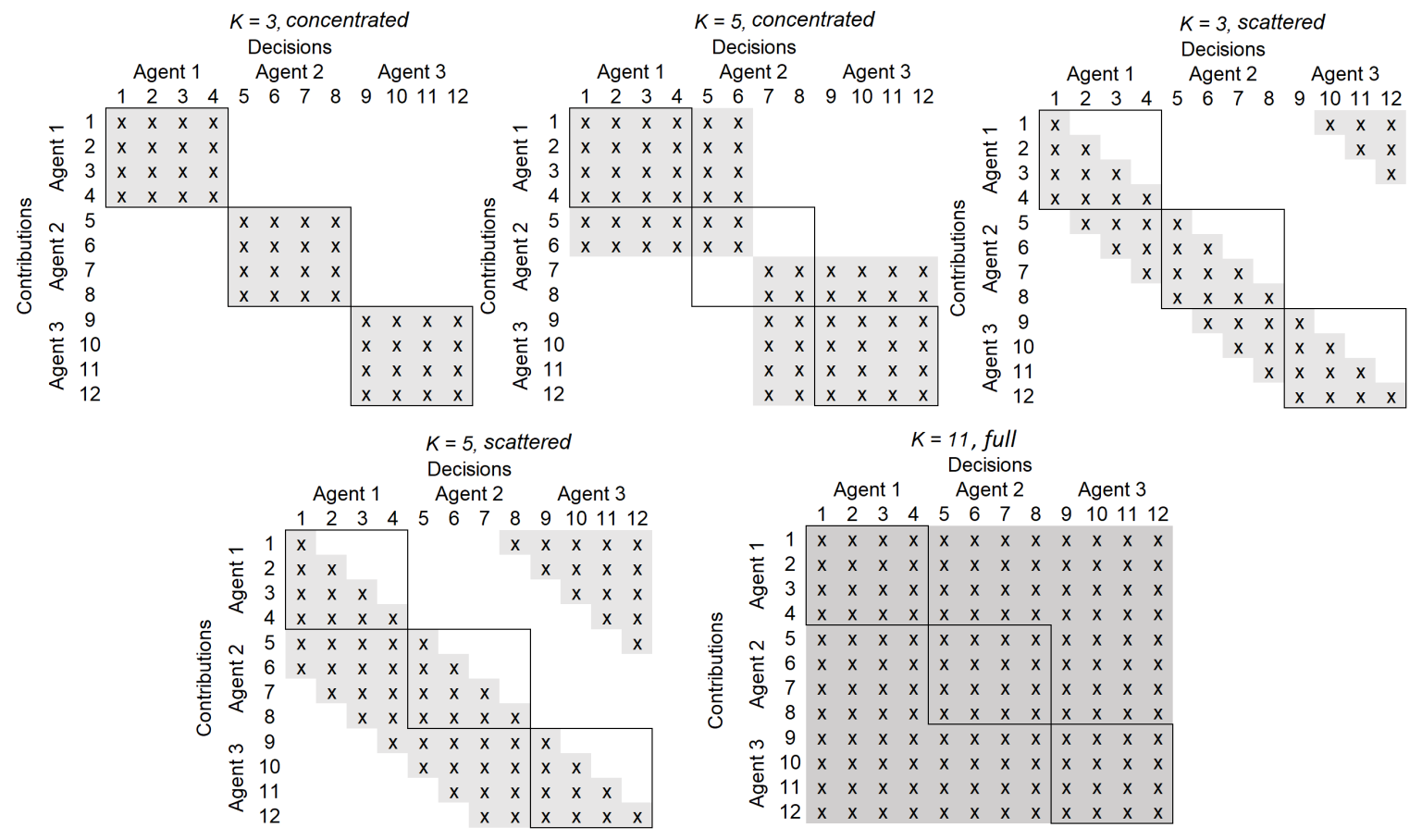

Figure 1: Matrices of inter-dependencies. ' $\mathrm{x}$ ' stands for inter-dependencies between decisions and performance contributions. Areas of expertise are indicated by solid lines.

Agents and individual adaptation Agents are part of one of the three areas of expertise. They are utility maximizers. Their utility is a weighted sum of their individual contribution to coalition performance $\frac{\sum_{\forall d_{i t} \in N_{s}} c_{i t}}{\left\|N_{s}\right\|}$ and the contribution of other agents to coalition performance $\frac{\sum_{\forall d_{i t} \in N_{-s} c_{i t}}}{\left\|N_{-s}\right\|}$, where $N_{\neg s}$ represents the decisions located outside the agent's area of expertise (i.e., the residual decisions). In the utility function of an agent $m$, these two parts are weighted by parameters $\alpha$ and $\beta$. The utility function is formalized in Eq. 2. Agents are myopic, since they determine their choices with the objective of maximizing utility in the current period without taking future utility into consideration.

$$
U_{m t}=\alpha \cdot \frac{\sum_{\forall d_{i t} \in N_{s}} c_{i t}}{\left\|N_{s}\right\|}+\beta \cdot \frac{\sum_{\forall d_{i t} \in N_{\neg s}} c_{i t}}{\left\|N_{\neg s}\right\|}
$$

At the beginning of each simulation round, agents are assigned to an area of expertise (i.e., to a partial binary decision sub-problem), and they are endowed with some capabilities in their area of expertise. This means that they initially know one of the $4^{2}$ possible solutions to the respective sub-problem. As agents do not have complete information about the solution space, they are boundedly rational (Simon 1957). Agents' capabilities might change over time, following a process of individual adaptation of capabilities. At every time step, agents can learn a new solution to their partial decision problem with probability $p$. This solution differs in one of the four values from the solution that they already know. In addition, with the same probability $p$, agents can forget a solution which they already know but which does not help in maximizing their utility in that particular time step. Regarding the adaptation of capabilities, we study three scenarios: 
- In the benchmark scenario we set $p=0$. Agents do not learn new or forget already known solutions to their partial decision problem.

- In the scenario with a low probability of adaptation, we set $p=0.2$

- In the scenario with a high probability of adaptation, we set $p=0.5$.

Coalitions and coalition formation As discussed above, self-organized coalition formation is modelled to follow a second-price auction. In second-price auctions, the top bidder wins and pays the second-highest bid (Vickrey 1961); agents are fully aware of the mechanism's functioning. Whenever an auction occurs, agents participate and bid the utility they expect from participating in a coalition (for an overview of similar approaches, see Rizk et al. (2019)). Since agents cannot observe the bids made by other agents or the solutions the other agents know, their expected utility is estimated by assuming that the residual decisions do not change compared to the previous period. Expected utilities $E\left(U_{m t}\right)$ are computed using the expected contribution of each decision to coalition performance, which is formalized in Eq. (3). The expected contribution of a decision is a function of the decision itself at time $t, d_{i t}$, the decisions within the area of expertise that are inter-dependent with the decision at time $t, \overline{\mathbf{d}}_{N_{s} t}$, and the residual decisions that are inter-dependent with the decision at time $t-1, \overline{\mathbf{d}}_{N_{\neg s} t-1}$.

$$
E\left(c_{i t}\right)=f\left(d_{i t} ; \overline{\mathbf{d}}_{N_{s} t} ; \overline{\mathbf{d}}_{N_{\neg s} t-1}\right)
$$

The expected utility function follows Eq. (2) and includes the agent's expectation about the contributions of individual decisions formalized in Eq. (3), so that

$$
E\left(U_{m t}\right)=\alpha \cdot \frac{\sum_{\forall d_{i t} \in N_{s}} E\left(c_{i t}\right)}{\left\|N_{s}\right\|}+\beta \cdot \frac{\sum_{\forall d_{i t-1} \in N_{\neg s}} E\left(c_{i t}\right)}{\left\|N_{\neg s}\right\|} .
$$

As the second-price auction assures that agents reveal their true preferences (Vickrey 1961), each agent bids the highest expected utility given the currently known subset of solutions. Once all agents have placed their bids, the top bidder for each area of expertise is determined. The top bidders of each area of expertise form the new coalition. Consequently, in our model, a coalition is always composed of three agents, i.e., one from each area of expertise.

Agents are given the possibility of collective adaptation every $\tau$ periods. Based on $\tau$, we distinguish three levels of instability.

- In the case of a stable coalition, a coalition is formed only once (in the very first time step), and there is no further opportunity for re-organization (i.e., $\tau=0$ ).

- In the case of a mid-stable coalition, there is the opportunity to reorganize the coalition every ten time steps (i.e., $\tau=10$ ).

- In the case of an unstable coalition, there is the opportunity to reorganize the coalition at every time step (i.e., $\tau=1$ ).

Once a coalition is self-organized, its task is to solve the complex decision problem introduced above. For simplicity, we assume that there is no further coordination or communication within a coalition nor outside of it.

Individual decision-making process and coalition strategy Agents are modelled to be utility maximizers. The agents who are part of the coalition are tasked with choosing a particular solution to their partial decision-making problem (i.e., in their area of expertise). This is done autonomously, with agents making their choices independently from each other. To make their choices, agents calculate the expected utility that each solution to their partial decision problem reports following Eq. (4). On this basis, each agent chooses the solution that promises the highest increase in expected utility (according to Eq. (4)). The overall strategy of the coalition is formed by concatenating the solutions chosen by the agents. Finally, the agents experience the resulting utility.

Process overview, scheduling, and main parameters The simulation model has been implemented in Python 3.7.4. Every simulation round starts with a preparation phase. The performance landscape is computed, and agents are randomly assigned to one area of expertise. They are given initial capabilities in the form of one initial solution to their partial decision-making problem. As there is no residual decision before the first time step, we compute a random $N$-dimensional bit-string as 
starting point in the performance landscape. After preparation has ended, the agents form a coalition in the first time step of each simulation run following the previously outlined procedure.

After the coalition is formed, each member of the coalition is tasked with choosing a solution in their area of expertise at every time step. The coalition strategy is formed by concatenating the solutions proposed by each agent in their particular area of expertise. Agents experience the resulting utility considering the particular coalition strategy and its associated performance. Finally, at the end of every time step, individual adaptation occurs according to the mechanism of individual adaptation previously described. After $\tau$ periods the agents are given the possibility to reorganize the coalition following the auction-based mechanism.

This process is repeated over $T$ time steps per simulation round. An overview of the process of the model is provided in Fig. 2.

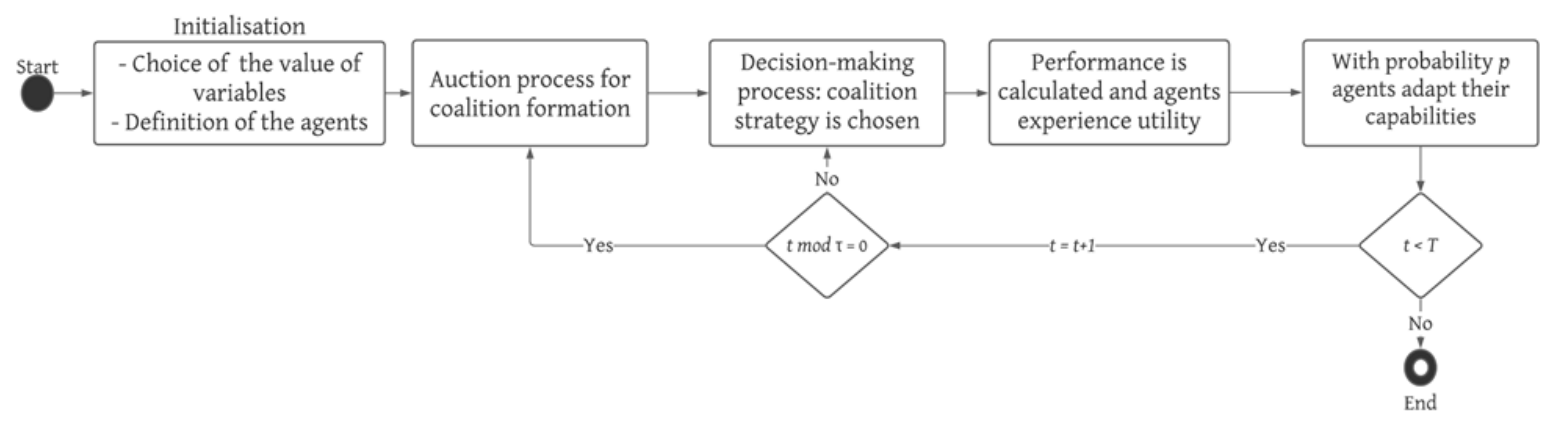

Figure 2: Process overview of the model. This figure represents the order in which every event occurs at a particular $t$

\section{SELECTED RESULTS}

\subsection{Scenarios and performance measures}

Table 1 summarizes the main variables and parameters used in our simulation study. Variables which are exogenously determined are (i) the level of inter-dependencies among sub-tasks $(K)$, (ii) the structure of inter-dependencies, (iii) the frequency of re-organization at the level of the coalition $(\tau)$, and (iv) the probability of individual adaptation/learning $(p)$. Given the ranges for these variables included in Table 1, we investigate 45 different scenarios. In each of these scenarios, we observe the results of the first 200 time steps because experiments have indicated that dynamics particularly emerge in this number of time steps. Based on the coefficient of variation, we fix the number of repetitions to 1,500.

Table 1: Main variables and parameters

\begin{tabular}{lllll} 
Factor & Type & Description & Denoted by & Ranges \\
\hline \multirow{6}{*}{ Variable } & Independent & Level of inter-dependencies & $K$ & $\{3 ; 5 ; 11\}$ \\
& Independent & Structure of inter-dependencies & Interaction matrix & $\{$ Concentrated; scattered; full $\}$ \\
& Independent & Frequency of re-organization & $\tau$ & $\{0 ; 1 ; 10\}$ \\
& Independent & Probability of individual adaptation & $p$ & $\{0 ; 0.2 ; 0.5\}$ \\
& Dependent & Coalition performance & $C\left(\mathbf{d}_{\mathbf{t}}\right)$ & $\in[0 ; 1]$ \\
\hline \multirow{2}{*}{ Parameter } & Temporal & Time step & $t$ & $\in[1 ; 200]$ \\
& Fixed & Time horizon & $T$ & $\{200\}$ \\
& Simulation & Simulation round & $r$ & $\in[1 ; 1,500]$ \\
& Fixed & Weights & $\alpha, \beta$ & $\{0.5\}$ \\
\hline
\end{tabular}

To assure that performances are comparable across scenarios, the observed coalition performance $C\left(\mathbf{d}_{\mathbf{t}}\right)$ achieved in a specific simulation round $r=1 \ldots, R$ at any time step $t=1, \ldots, T$ is normalized by the maximum performance which is achievable on the landscape on which the simulation run is performed, $\max (C)$. The normalized coalition performance at every time step $t$ is averaged across the 1,500 simulation rounds of each scenario, resulting in the normalized average performance

$$
\tilde{C}_{t}=\frac{1}{R} \sum_{t=1}^{T} \frac{C\left(\mathbf{d}_{\mathbf{t}}\right)}{\max (C)} .
$$


We report the sum of the Manhattan Distance for each scenario, i.e., the distance between the normalized average coalition performance at each time step and the best performance attainable in that particular scenario (which, after normalization, is equal to 1). This allows us to report a measure that reflects, on average, how each scenario performs. We compute this performance measure according to

$$
D=\sum_{t=1}^{T}\left(1-\tilde{C}_{t}\right)
$$

In Fig. 3, we plot the Manhattan Distance for all scenarios resulting from the parameters included in Table 1. Each sub-plot represents the results for a different level of individual adaptation $(p=0$ in contour plot $1, p=0.2$ in contour plot 2 , and $p=0.5$ in contour plot 3 ). The horizontal axes provide information about the complexity of the task environment (in terms of the level of inter-dependencies $K$ and their structure). Within each contour plot, complexity increases from the left to the right. The vertical axes provide information about the frequency of coalition formation $(\tau)$; this frequency increases from the bottom to the top. Note that the performance measure plotted in Fig. 3 is the distance between the average performance achieved by a coalition and the maximum performance. Lower values, thus, indicate better performing coalitions.

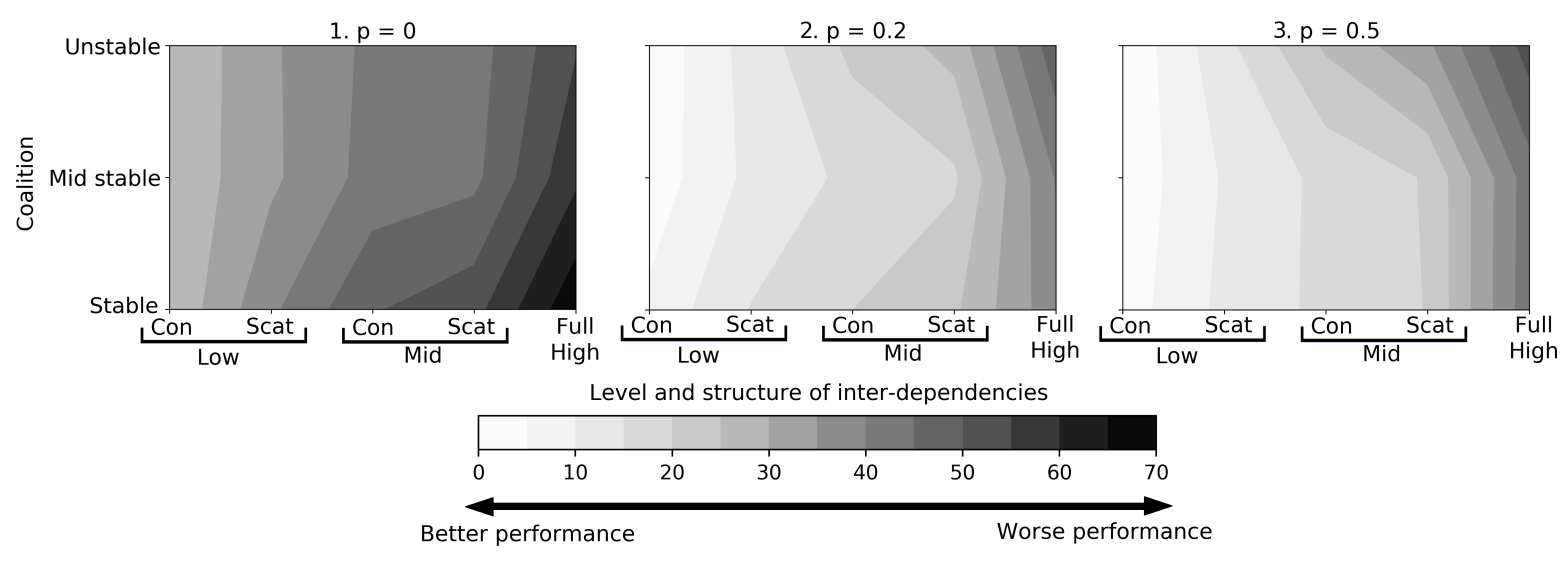

Figure 3: Contour plot. Contours are based on the Manhattan Distance (see Eq. (6)). Note: Con refers to a concentrated structure and Scat to a scattered structure.

\subsection{Results}

Results indicate that there exists a negative relationship between the level of inter-dependencies $(K)$ and the performance achieved by a coalition: We can observe that increases in complexity lead to a decrease in coalition performance. If we move further to the right on the horizontal axis of the contour plots presented in Fig. 3, the Manhattan Distance increases, indicating that the distance between the maximum performance and the achieved performance increases. For $p=0.2$, for instance, moving from low inter-dependencies $(K=3)$ and a concentrated structure to full inter-dependencies $(K=11)$ increases the Manhattan Distance from a value of less than 6 to a value of more than 40. This observation is robust across all frequencies of re-organization (i.e., all values of $\tau$ ) and all considered probabilities of individual adaptation (i.e., all values of $p$ ).

In addition to the observations related to the level of inter-dependencies $(K)$ on performance discussed above, the results suggest the following effect for the structure of inter-dependencies: Performances tend to decrease when we move from a concentrated to a scattered structure within one level of interdependencies. This observation is particularly pronounced for low levels of complexity and robust across all probabilities of individual adaptation (i.e., $p$ ) and all frequencies of re-organization at the level of the coalition (i.e., $\tau$ ). For instance, moving from the concentrated to the scattered structure for mid-stable coalitions on the left-hand side of contour plot 2 in Fig. 3 (i.e., the case of low interdependencies $(K=3)$, a moderate probability of individual adaptation $(p=0.2)$, and a high frequency of re-organization $(\tau=10)$ ), increases the Manhattan Distance from a value below 1.84 to a value beyond 11.33. That is an increase of 6 times in value. For a medium level of inter-dependencies $(K=5)$ 
and the same values of $p=0.2$ and $\tau=10$, moving from the concentrated to the scattered structure leads to a relatively smaller increase in the Manhattan Distance from a value of 16.16 to a value of 19.20. This observation might be explained by the fact that higher levels of inter-dependencies do no longer allow for a fully self-contained structure, even in the concentrated case. This means that for high levels of $K$, the inter-dependencies cannot be completely located within an area of expertise (see Fig. 1). In other words, the marginal decrease in coalition performance is relatively low when the number of inter-dependencies across areas of expertise is already relatively high.

Results suggest that individual adaptation $(p)$ - i.e., the capability to learn - is a key factor for performance. Coalition performance improves considerably when we move from $p=0$ to $p=0.2$ or $p=0.5$ (see Fig. 3). For the case of no individual adaptation $(p=0)$, low levels of inter-dependencies $(K=3)$, a concentrated structure, and a mid-stable coalition $(\tau=10)$, endowing the agents with the capability to learn with a probability of $20 \%$ (i.e., moving from $p=0$ to $p=0.2$ ) decreases the distance between the performance achieved by this coalition and the maximum attainable performance from a value of 25.90 to a value of 2.82. Performance, thus, increases to a large extent when agents are endowed with learning capabilities. For higher levels of complexity (i.e., for higher values of $K$ ), the same effect can be observed, even though it is less pronounced. For $K=11$ and $\tau=1$, moving from $p=0$ to $p=0.2$ decreases the distance only from a value of 43.69 to a value of 40.34 . For cases in which agents are already endowed with learning capabilities, increasing the probability of individual adaptation $p$-i.e., increasing the frequency of learning - only has a significant effect on performance when the complexity of the task environment is considerably high. This indicates that the positive effect of individual adaptation is only relatively high if non-adapting $(p=0)$ agents learn to adapt to the environment $(p>0)$. For agents who are already well-endowed with the capability of individual adaptation (in terms of a high $p$ ), the effect is non-significant. In other words, the marginal positive effect of endowing agents with the capability to adapt to the environment - in terms of learning decreases with $p$.

Concerning the frequency of adaptation at the level of the coalition $(\tau)$, the results suggest a strong interaction between $\tau$ and the probability of individual adaptation. For high-levels of inter-dependencies ( $K=5$ and $K=11$ ), the pattern in the contour plots presented in Fig. 3 appears to be substantially shaped by the probability of individual adaptation in the following way: For coalitions which are composed of agents who are not endowed with learning capabilities (i.e., $p=0$, see contour plot 1 in Fig. 3), a frequent re-organization of the coalition appears to be beneficial for coalition performance. For coalitions which are composed of agents who learn with moderate probability (i.e., $p=0.2$, see contour plot 2 in the middle of Fig. 3), re-organization appears not to play a central role when it comes to performance. If we move to coalitions which are composed of individuals who learn very frequently (i.e., $p=0.5$, see contour plot 3 in Fig. 3), the pattern appears to shift into the opposite direction, so that stability in the composition of a coalition tends to have positive effects on performance. If coalitions face less complex tasks (i.e., if we move to the left on the horizontal axes in the contour plots presented in Fig. 3), this pattern becomes insignificant, so that the effect of the frequency of re-organization at the coalition level no longer has effects on performance. This observation might be explained by the fact that both individual adaptation and re-organization at the coalition level have similar consequences from the coalition's perspective. The former allows the agent to find new solutions to his or her partial decision problem which are, then, contributed to solving the problem which the entire coalition faces. The latter, by replacing the members of a coalition, can introduce new members who might bring in new solutions to solve the decision-making problem the coalition faces. From the perspective of the coalition, both individual and collective adaptation foster the innovativeness of the coalition: In case of agents who are not endowed with learning capabilities (i.e., $p=0$ ), a coalition assures innovativeness by frequently replacing members. For coalitions already composed of agents who often learn and make progress (i.e., $p=0.5$ ), there is no need to replace members, since the coalition is already quite innovative. Our results indicate that too much innovativeness in the above sense is detrimental to coalition performance. In other words, if a coalition which already comes up with innovative ideas faces a complex problem, one would be well-advised to assure that the coalition does not re-organize and replace members - this perfectly translates to "never change a winning team", at least do not do so when this team faces a very complex task. 


\section{CONCLUSION}

\subsection{Summary}

From on the results presented in Sec. 4, four key findings can be derived. First, there is a negative relationship between the level of inter-dependencies among sub-tasks of a complex decision-problem $(K)$, which is faced by a coalition, and the achieved performance. This finding is in line with previous literature, which finds that a higher degree of complexity leads to a larger number of local maxima (e.g., more rugged landscapes in the context of the $N K$-framework) and, consequently, a higher chance of getting stuck at a local maximum (Levinthal 1997, Leitner and Wall 2014).

Second, the structure of inter-dependencies among sub-tasks of a complex decision-problem appears to have substantial effects on performance at moderate levels of complexity. For complex decision problems, though, the impact of the structure of inter-dependencies is negligible. This finding is also in line with the results of previous studies on task allocation in complex environments, such as Wall (2018) and Hsu et al. (2016).

Third, endowing decision-makers with learning capabilities, so that the probability of adapting to the environment increases, is particularly fruitful for agents who just learn to adapt. For agents who are already well-trained in adapting to the environment, marginal positive effects decrease significantly. Results of the $N K$-model suggest that the positive effect of coming up with new solutions is relevant at the beginning of the simulation, but its relevance decreases over time (Levinthal 1997). This could explain why the marginal positive effects of adaptation diminish as $p$ increases: Gains coming from individual adaptation would only be relevant for the first time steps.

Finally, whether adaptation at the coalition level is beneficial for performance is strongly affected by the characteristics of the members who form the coalition. While innovative coalitions that face complex decision problems should make every effort not to replace members, less innovative groups would be well-advised to replace members in a self-organized manner to increase performance. If the faced decision problem is of low (or in some cases moderate) complexity, the effect of (in)stability becomes insignificant. This is in line with the results of Wall (2018) and Hsu et al. (2016).

\subsection{Conclusive remarks}

This research is a first attempt to systematically integrate the dynamic capabilities framework into problems of coalition formation in complex task environments. Results are consistent with and extend the main insights from previous literature, such as Leitner and Wall (2014), Levinthal (1997) and Wall (2018). Our research is, however, not without its limitations. First, we consider human decisionmakers who are rational in the sense of perfect foresight when evaluating the performance on their landscape (i.e., they make no errors when predicting expected utilities) (Hendry 2002), they do not suffer from decision making biases (Kahneman et al. 1982), and they can correctly handle complex decision problems (i.e., they have the cognitive capacity to handle decision problems irrespective of their complexity) (Pennington and Hastie 1986). Moreover, we do not consider coordination mechanisms within coalitions; in our model, every agent acts in a fully autonomous manner. Future research might want to include mechanisms to coordinate decisions within a coalition, such as suggested in Wall (2018). Our research can also be expanded by introducing heterogeneity in the adaptation of capabilities or shocks that change the shape of the solution space. All these features could lead to new insights within the contexts of complex decision-making and dynamic coalition formation.

\section{ACKNOWLEDGMENTS}

We would like to thank Joop van de Heijning and Ravshanbek Khodzhimatov (University of Klagenfurt, Austria) for their valuable assistance in the implementation of the model.

\section{REFERENCES}

Banerjee, S., H. Konishi, and T. Sönmez. 2001. "Core in a simple coalition formation game”. Social Choice and Welfare 18 (1): 135-153.

Eisenhardt, K. M., and J. A. Martin. 2000. “Dynamic capabilities: What are they?". Strategic Management Journal 21:1105-1121. 
Giannoccaro, I., G. Massari, and G. Carbone. 2018. "Team resilience in complex and turbulent environments: The effect of size and density of social interactions". Complexity 2018 (6): 1-11.

Hendry, J. 2002. "The principal's other problems: honest imcompetence and the specification of objectives". Academy of Management Review 27 (1): 98-113.

Hennet, J. C., and S. Mahjoub. 2011. "Coalitions of firms in manufacturing networks: Stability and optimality issues". In Proceedings of the 18th World Congress The International Federation of Automatic Control, Milano, 6419-6424. IFAC.

Hsu, S. C., K. W. Weng, Q. Cui, and W. Rand. 2016. "Understanding the complexity of project team member selection through agent-based modeling". International Journal of Project Management 34 (1): 82-93.

Kahneman, D., P. Slovic, and A. Tversky. 1982. Judgment under uncertainty: Heuristics and biases. Cambridge: Cambridge University Press.

Leitner, S., and F. Wall. 2014. "Multiobjective decision making policies and coordination mechanisms in hierarchical organizations: Results of an agent-based simulation". The Scientific World Journal 7:112.

Leitner, S., and F. Wall. 2015. "Simulation-based research in management accounting and control: an illustrative overview". Journal of Management Control 26 (2-3): 105-129.

Levinthal, D. A. 1997. "Adaptation on rugged landscapes". Management Science 43 (7): 934-950.

İnal, H. 2019. "The existence of a unique core partition in coalition formation games". Games and Economic Behavior 114:215-231.

Pennington, N., and R. Hastie. 1986. "Evidence evaluation in complex decision making.". Journal of personality and social psychology 51 (2): 242-258.

Rivkin, J. W., and N. Siggelkow. 2003. "Balancing search and stability: Interdependencies among elements of organizational design". Management Science 49 (3): 290-311.

Rizk, Y., M. Awad, and E. W. Tunstel. 2019. "Cooperative heterogeneous multi-robot systems: A survey”. ACM Computing Surveys 52 (2): 29:1-29:31.

Simon, H. A. 1957. Models of man, social and rational. John Wiley and Sons.

Sless, L., N. Hazon, S. Kraus, and M. Wooldridge. 2018. "Forming k coalitions and facilitating relationships in social networks". Artificial Intelligence 259:217-245.

Teece, D. J., G. Pisano, and A. Shuen. 1997. "Dynamic capabilities and strategic management: organizing for innovation and growth”. Strategic Management Journal 18 (7): 509-533.

Tremewan, J., and C. Vanberg. 2016. "The dynamics of coalition formation - A multilateral bargaining experiment with free timing of moves". Journal of Economic Behavior and Organization 130:33-46.

Vickrey, W. 1961. "Counterspeculation, auctions, and competitive sealed tenders". The Journal of Finance 16 (1): 8-37.

Wall, F. 2018. "Emergence of task formation in organizations: Balancing units' competence and capacity". Journal of Artificial Societies and Social Simulation 21 (2): 1-26.

Wall, F., and S. Leitner. 2020. "Agent-based computational economics in management accounting research: Opportunities and difficulties". Journal of Management Accounting Research (11).

\section{AUTHOR BIOGRAPHIES}

DARIO BLANCO-FERNÁNDEZ is a PhD student in the fields of Social Sciences and Economics at the University of Klagenfurt. He is currently in his second year. His email address is dario.blanco@aau.at and his ORCID ID is 0000-0002-8275-701X

STEPHAN LEITNER is an Associate Professor at the Department of Management Control and Strategic Management at the University of Klagenfurt. His email address is stephan.leitner@aau.at and his ORCID ID is 0000-0001-6790-4651

ALEXANDRA RAUSCH is an Associate Professor at the Department of Management Control and Strategic Management at the University of Klagenfurt. Her email address is alexandra.rausch@aau.at and her ORCID ID is 0000-0002-9275-252X 